\title{
[ARTI GO RETRATADO] Análise do equilíbrio no processo de envelhecimento
}

\section{Analysis of the balance in the aging process}

\section{Larissa Lomeu de Figueiredo(I); Tatiane da Silva Pícoli(I I); Ana Paula Oliveira Borges( I I ); Lislei Jorge Patrizzi( I V)}

(I) Especialista em Geriatria pela Universidade Estadual de Campinas (Unicamp), PósGraduanda em Fisioterapia Ortopédica, Traumatológica e Desportiva pela Pontifícia Universidade Católica de Minas Gerais (PUC Minas), Poços de Caldas, MG - Brasil, e-mail: larissalf.fisio@gmail.com

(II) Pós-Graduada em Fisioterapia Cardiorrespiratória pela Universidade de Franca (Unifran), Franca, SP - Brasil, e-mail: tatianepicoli@yahoo.com.br

(III) Docente do curso de Fisioterapia da Universidade de Franca (Unifran), Franca, SP - Brasil, e-mail: anaproliveira@uai.com.br

(IV) Professora adjunta do curso de Fisioterapia da Universidade Federal do Triângulo Mineiro (UFTM), Uberaba, MG - Brasil, e-mail: Ipatrizzi@uol.com.br

A revista Fisioterapia em Movimento comunica a retirada do artigo Análise do equilíbrio no processo de envelhecimento. Analysis of the balance in the aging process. Larissa Lomeu de Figueiredo, Tatiane Picoli; Ana Paula Oliveira Borges; Lislei J orge Patrizzi. Fisioter Mov. 2011 jul/ set;24(3):401-7 por motivo de plagio no tópico discussão. Os autores admitem a falha ética e apresentam suas sinceras desculpas. Abaixo as fontes originais:

1. ALTERAÇÕES NO FUNCIONAMENTO DO SISTEMA DE CONTROLE POSTURAL DE IDOSOS: USO DA INFORMAÇÃO VISUAL. Paulo Freitas J únior; J osé A. Barela. Rev Port. Cien Desp. 2006. 6(1) 94-95

2. CARACTERÍSTICAS COMPORTAMENTAIS DO CONTROLE POSTURAL DE J OVENS, ADULTOS E IDOSOS. Paulo Barbosa de Freitas J unior. Unesp, Rio Claro SP. 2003.

Profa. Dra. Auristela Duarte de Lima Moser Editora Chefe 


\section{Análise do equilíbrio no processo de envelhecimenı}

\section{Analysis of the balance in the aging process}

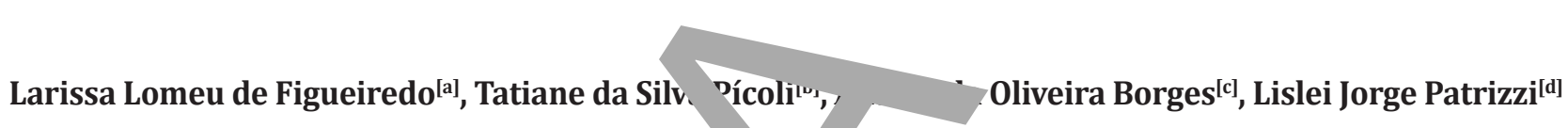

[a] Especialista em Geriatria pela Universidade Estadual de ( nas (Unicamp), Pós-Graduanda em Fisioterapia Ortopédica, Traumatológica e Desportiva pela Pontifícimiversidade ‘́lica de Minas Gerais (PUC Minas), Poços de Caldas, MG Brasil, e-mail: larissalf.fisio@gmail.com

[b] Pós-Graduada em Fisioterapia Cardic spiratór pola Univers ade de Franca (Unifran), Franca, SP - Brasil, e-mail: tatianepicoli@yahoo.com.br

[c] Docente do curso de Fisioterapia nnersida de Franca,_nifran), Franca, SP - Brasil, e-mail: anaproliveira@uai.com.br

[d] Professora adjunta do curso de Fisı via Jniversidade Federal do Triângulo Mineiro (UFTM), Uberaba, MG - Brasil, e-mail: Ipatrizzi@uol.com.br

\section{Resumo}

Introdv o: Durante o processo ¿é envelhecimento, nota-se uma diminuição das reservas funcionais e fisiológica 10 organi mo. Essas alterações favorecem a diminuição das habilidades dos idosos em executarem alg' as tarefas in que muitas vezes o simples ato de equilibrar-se se torna um desafio. Objetivos: Avaliar o e. 'hrio fur snal no pr esso de envelhecimento. Materiais e métodos: Foram avaliadas 48 pessoas com idade 85 ano ambos os sexos, divididas em 4 grupos de acordo com a idade (contendo em cada orupo $12 \mathrm{lh} \quad$ uos, de 6 homens e 6 mulheres), sendo: grupo 1 (GI) 10-18; grupo 2 (GII) 20-35; grupo 3

45-60; gru (GIV) 65-85. Os instrumentos de avaliação utilizados envolveram a escala de equilíbrio de $\mathrm{E}$ B. a avaliação da marcha e equilíbrio orientada pelo desempenho (POMA), o teste de apoio unipo , o tes. alcance funcional e o teste de Romberg. Resultados: Mostrou-se que pelo menos um grupo ) se difere, ao nível de $5 \%$ de significância, em todas as variáveis estudadas. Conclusão: Sugere-se que o rocesso de envelhecimento altera o equilíbrio funcional com maior intensidade a partir dos 62 anos de idade.

Pa. S-chave: Equilíbrio postural. Envelhecimento. Idoso. 
Abstract

Introduction: While growing elder, it is possible to notice a dicrease in the functiona in the organism witch impairs the decrease of the elderly abilities doing some tasks a challenge. Objectives: evaluate the functional balance in the aging process. Mat evaluated 48 people from 10 to 85 years old, os both sexes. Divided in 4 groups acoor $g$ to the age (each with 12 individuals, being 6 men and 6 women), being: group 1 (GI) 10-18; group 2 ( 20-35; group (GIII) 45-60; group 4 (GIV) 65-85. The evaluation instruments are the Berg balanro scale (L. rmance oriented mobility assessment (POMA), the unipodal support test, the fur snat r +est and the Romberg's test. Results: Show that al least one group (G4) difers 5\% in every varir estudied. Con suggests that the aging process modifies the functional balance with gger intensity from e 62 years of age.

Keywords: Postural balance. Aging. Aged.

\section{Introdução}

O envelhecimento é um processo dinâmico e progressivo. Com o aumento da idade cronológica, as pessoas tornam-se menos ativas, suas capacidad físicas diminuem e as alterações psicológicas q e acompanham a idade, como sentimento de velhice, estresse e depressão, desmotivam a prática de exercícios, facilitando o aparecimento de doen cas que aceleram o processo de envelhecime (1).

O sedentarismo, a incapacidade e a depend ia são grandes adversidades à saúde, que, associac ao envelhecimento, contribuem pa da da au tonomia e maior risco de instituci alizaça De acordo com o Centro Nacional de Es ustica pa aproximadamente $84 \%$ das $\mathrm{r}$ nas com ade iguà ou superior a 65 anos são depen suas atividades cotidian Estima-so em 2020 ocorrerá aumento de \% a $167 \%$ no m ro de idosos com moderad ove incapacidade (2).

Um dos princi s fator ue limitam hoje a vida do idoso é o desequilíbrio. ᄂ $\quad 0 \%$ dos casos, esse fato não $\mathrm{p}$ é ser atribuído a uma causa específica, mas sim am comrometimento do sistema de equilíbrio o no um to . Em mais da metade dos casos, o de vilíbric m origer entre os 65 e os 75 anos aproxin. ente e ce de $30 \%$ dos idosos apresentam os Sir nas $\mathrm{r}$ se período. As quedas sãc ocu quências perigosas do desequilíb $\Omega$, são re náveis por $70 \%$ das mortes acidenis em pe Jas cu ais de 75 anos (3).

O ea abrio corporal é definido como a manuten ş uma postura particular do corpo com um mínimo ccilação (equilíbrio estático) ou a ma-

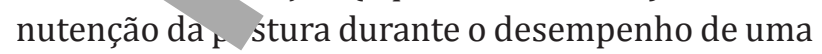

habilidade otor que tenda a perturbar a orientac) do corpc ailíbrio dinâmico) (4). Na maioria as vezes, pens os em equilíbrio somente em ocaiões especiais, c 0 manter-se em postura unipou. aminhar sobre uma superfície estreita ou piso molha Embora essas tarefas exijam equilíbrio, os mecanı s envolvidos no controle postural são requeridos em atividades simples, como caminhar, levantar-se, mudar de direção e subir escadas, situsentes no cotidiano (5).

Estima-se que a prevalência de queixas de equilíbrio na população acima de 65 anos chegue a 85\%, estando associada a várias etiologias, tais como: degeeração do sistema vestibular, da capacidade visual e da perseguição uniforme; alterações proprioceptivas; déficits musculares esqueléticos (sarcopenia); hipotensão postural; atrofia cerebelar; diminuição do mecanismo de atenção e tempo de reação, contribuindo para alterações do equilíbrio na habilidade de executar as atividades da vida diária (AVDs) (6-11).

A queixa de dificuldade de equilíbrio e marcha, assim como as histórias prévias de quedas, têm sido apontadas como fatores de risco para idosos que vivem em comunidade (12). Com etiologia multifatorial, ocorrem graças à combinação de fatores intrínsecos (idade, déficit cognitivo, fraqueza muscular, hipotensão postural, deficiência visual, déficits do sistema vestibular, anormalidades da marcha e equilíbrio, problemas nos pés e medicações) e extrínsecos (comportamentos e atividades do indivíduo e seu meio ambiente) (6-8).

Dessa forma, como o equilíbrio sofre alterações durante o processo de envelhecimento, propiciando a alteração da marcha e o surgimento de quedas, o 
objetivo deste estudo foi avaliar o equilíbrio funcional no processo de envelhecimento.

\section{Materiais e métodos}

\section{Sujeitos}

Os participantes foram selecionados nas dependências da Universidade de Franca (Unifran) e nos domicílios do bairro. A escolha dos grupos foi baseada de acordo com o grau de funcionalidade e independência, tendo em vista analisar o déficit de equilíbrio no processo de envelhecimento saudável. Dessa forma, o critério de seleção dos participantes foi a ausência de patologia de base que interferisse diretamente no equilíbrio.

Os critérios de exclusão foram: alteração neurológica, déficit cognitivo, lesão ortopédica, fazer uso de órteses para membro inferior, obesidade, fazer exercício com frequência superior a duas vezes na semaná e ser portador de qualquer tipo de comprometimento para a sua permanência na posição ortostática.

Foram escolhidos 48 indivíduos de ambos xos e com idades compreendidas entre 10 e 85 os (média 40,31 + 23,38 anos). Esses indivíduos for. divididos, de acordo com a idade, em quatro grupos (contendo em cada grupo 12 indiví endo 6 homens e 6 mulheres): grupo I (G 10-1乞 jrupo II (G2): 20-35; grupo III (G3): 45- ; Grupo 65-85.

Este estudo foi aprovado nelo $u$ it ética e Pesquisa dessa instituição

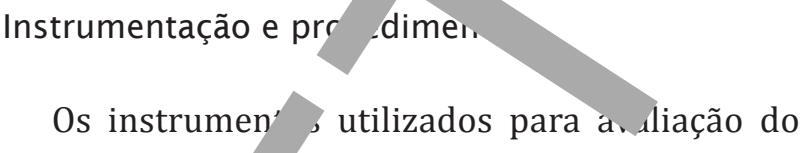
equilíbrio forar

1) escal Berg: nposta pr 14 itens com atividades a da vida ária, com grau de dificuldade pr ssivo ada item é graduado u- ontos e a ção da escala dura em média : inutos e requer uma régua e um relógir 13,14

avali do da marcha e equilíbrio orientada pelo npenho (POMA): consiste em um protocolo dido em duas partes, que totalizam 28 pontos, avaliando-se o equilíbrio estático e dinâmico, com manobras semelhantes a atividades da vida diária;

3) teste de apoio unipodal: paciente deve ilibrar-se durante $30 \mathrm{se}$ indos em um pe ó olhando fixamente em $\mathrm{u}$ ponto fixo a $2 \mathrm{~m}$ e distância. $\mathrm{O}$ cronômetro $t$ rado no prim $\mathrm{O}$ contato com o chão.

4) teste de alcanc ailciun. daptado: que avalia a estabili de na posiça ortostática. Os participan , permaneciam pé, projetavam o corpo frente o máx 10 possível ao longo de uma fita 'trica co' ada na parede próximo aos paciente. ara do acrômio;

5) $\mathrm{Te}$ mherg e teste de Romberg-Barré ou h berg sc do: realizado em solo estáve ins ${ }^{\dagger}$ el, e todas as posições foram realizada meiro de olhos abertos e depois de olhos fe dos. A duração de cada posição foi de 30 seg los, com posicionamento dirente dos pés.

Os testes lcance funcional e apoio unipodal foram solicitados três vezes, sendo o resultado a média dos valores obtidos.

la posicionamento dos pés, foi estabeleda uma graduação de 0 a 4 (sendo 0 - ausência de comprometimento, e grau 4 - maior compromeimento) de acordo com o grau de dificuldade dos t icionamentos solicitados.

\section{Análise estatística}

Inicialmente foi realizada uma análise descritiva dos dados obtidos, sendo avaliada a distribuição normal das variáveis estudadas. Os dados que não apresentaram distribuição normal foram transformados (Teste de Johnson) para então ser aplicado o tTeste ANOVA. Para as variáveis que não apresentaram distribuição normal após transformação foi utilizado o teste de Kruskal-Wallis (teste não paramétrico). Para as conclusões das análises estatísticas foi considerado o nível de significância de $5 \%(\mathrm{p}<0,05)$.

\section{Resultados}

Os resultados mostram que pelo menos um grupo (G4) se difere ao nível de 5\% de significância em todas as variáveis estudadas, sugerindo por meio dos 
valores obtidos maior comprometimento funcional neste grupo (Tabelas 1 e 2).

\section{Discussão}

Há um consenso de que os idosos diminuem sua capacidade de controle postural. Alguns estudos têm apontado que os idosos apresentam alterações comportamentais durante a manutenção da postura ereta (15-18). Tem sido sugerido que as causas da diminuição no desempenho do controle postural em idosos estariam associadas às alterações estruturais e funcionais nos sistemas sensoriais e motor e a problemas na integração das informações sensoriais $(19,20)$. Foi verificado frequentemente que os idosos oscilam mais que os adultos jovens, tanto de olhos abertos quanto de olhos fechados $(19,21-24)$.

Em geral, os estudiosos sugerem que o aumento nas oscilações corporais em idosos seria consider? do um indício de alterações no sistema de contr postural. Segundo Wolfson et al. (25) as diferenças observadas entre idosos e ad $\_$auran. manutenção da postura ereta, em n primeiro mo ento, não seriam causadas pela lterações estru ais dos sistemas sensoriais que correm em funç do processo natural de envelhe ento, mas es riam associadas a alterações natológ desses sistemas. $Y$ ciltan s dados acima citados não condizf com os resu dos do presente estudo, pois $\mathrm{n}$ foram estabele los critérios de exclusão, evitan narticipação indivíduos com patologias.

Além das alterações su $r$ s e motoras verificadas nco da idade, us alterações no sistema ner o, tais c. ninuição na velocidade de transm ão $d$ impulso nervoso nos neurônios s soriais e ores $(26,27)$; a perda significativa e neurônios, c. 'endritos e redução no número de "amificações ner as que prejudicam a comunicaç. ntre as células nervosas; a diminuição do metaboh cerebral; a redução da perfusão cerebral

Tabela 1 - Dados referentes à idade, escala de equ rio de be funcional (AF), desvio-padrão (DP) e p-valo 'esu' dos dos testes

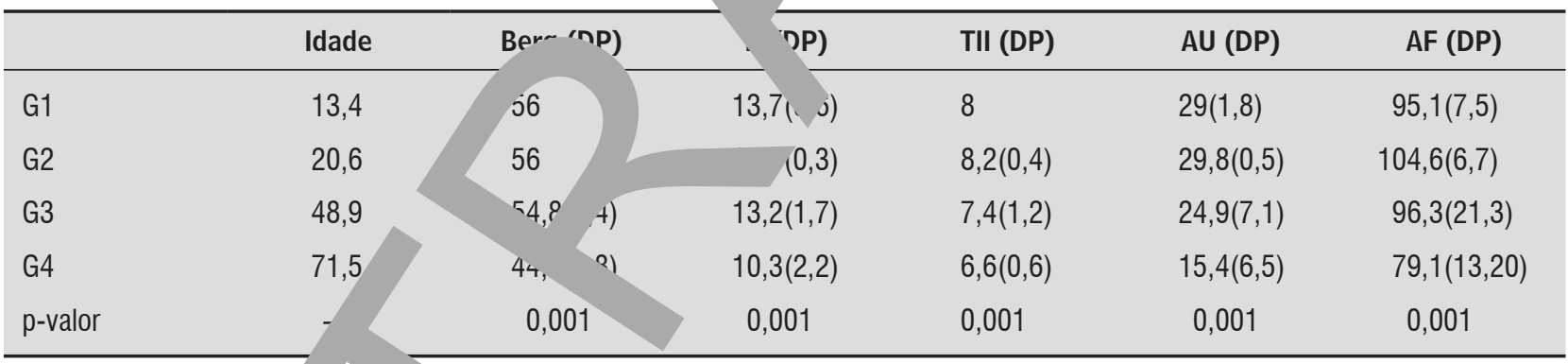

Fonte: Dados da pesquic

Tabela 2 - Rr serg olho abertos e fechados solo estável (ROAE, ROFE); Romberg olhos abertos, fechados solo instável JAI), des' padrão (DP) e p-valor. Resultados dos testes de Romberg

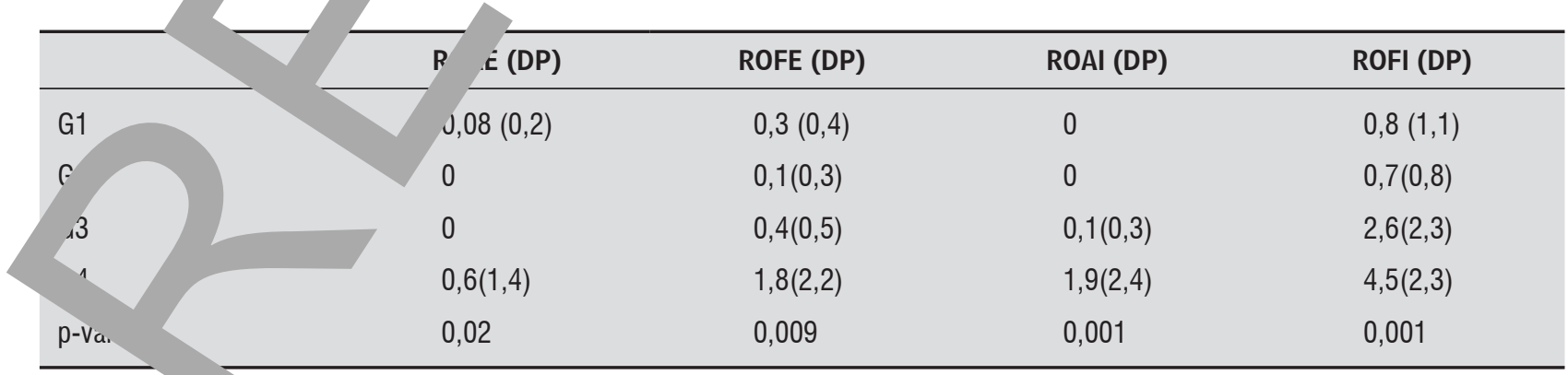

Fonte: Dados da ${ }_{\text {, squisa. }}$ 
e alteração no metabolismo dos neurotransmissores (28), poderiam interferir no desempenho do sistema de controle postural. A diminuição na velocidade de transmissão do impulso nervoso pelas vias aferentes e eferentes pode afetar consideravelmente $o$ sistema de controle postural, principalmente em situações de perturbação, em que o tempo necessário para desencadear uma resposta postural é imprescindível para o sucesso da recuperação do equilíbrio. Esse problema seria o aumento no tempo necessário para o processamento da informação e para o início da resposta motora. Comportamentalmente, segundo Spirduso (29), os idosos apresentam um maior tempo de reação (TR) comparado ao dos adultos e esse tempo de reação tem um aumento quando a tarefa envolve maior processamento de informação (TR de escolha e TR discriminativo).

Esses dados confirmam os resultados obtidos no presente estudo, no qual foi observado maior nível de comprometimento de equilíbrio funcional no grupo de idosos quando comparado com o de adoles centes, jovens e adultos.

Além das alterações sensoriais, o processo de envelhecimento também causa mudanças estr e funcionais no sistema neuromuscular (30 ?). Essas mudanças estruturais provocam, basicamı te, uma diminuição dos níveis de força e um aumen to no tempo para a produção de forc ma (18, $30,32,33)$. Somando-se às alteraçõ acima tadas, os idosos apresentam uma redur na elast do tecido conectivo muscular. 'iminuic da capacidade elástica do músculo somc. ì iudanças estruturais e funcionais de articulaço inoviais, podem levar os idosos a resentar menor o de flexibilidade e, consec .. nente, menor amplitude de movimento cicular Com relação à amplitude de movimento articular, ${ }_{1}$ 's-se afirmar que a amplitude movimento nas artı.ulações do tornozelo, joelb e quadril necessária para a manutenção da pos ra ereta mínima. Durante a manutenção da $\mathrm{F}$ ura ere , os deslr mentos angulares do tornozè o e quar não ultrapassam 4 graus (24). Desse do, a dução na amplitude de $m$ nas artic. ses do tornozelo, joelho quadril in óm não seria um fator determin ce para o sntro stural em idosos. Gu e cola. dor (35) observaram que o valor máximo de tor r. rticular do tornozelo gerado por idosos durante pe. hações do equilíbrio foi maior que o torque gerado $\mathrm{p}$ os adultos, ficando ao redor de 20
N.m em média. Segundo o mesmo autar esse valor é muito inferior ao valor de torc maximm tornozelo que os idosos podem oduzir. Com es resultados, os autores concluí m que, se os ido s apresentam dificuldades em ntrolar a postu?, essa dificuldade não está rela nada à prod de torque articular e sim a uma força muscular redur a. Lu mo modo, Maki e Mcllroy (28) tamb a afirmaram a magnitude de força muscula equerida para a mutenção da postura ereta e par. sencadear um passada após uma perturbação ao eq. rio é $m$ o menor que a capacidade dos idosos em gu f ça, ou seja, mesmo com icão na capacruade de gerar força, os idos consegu força muscular suficiente para 1 nter em postura ereta, e mesmo par responde erturbações utilizando outras e atégias de co ole. A partir desses resultados, der-se-ia sugerir a capacidade de gerar força mus $\operatorname{c}$ não afetaria significativamente o controle posturà josos.

Dessa for o equilíbrio corporal é alcançado quando todas as forças que agem nesse corpo, tanto nternas como internas estão controladas, o que perI) corpo permaneça em uma posição dejada (equilíbrio estático) ou que se mova de uma maneira controlada (equilíbrio dinâmico). Esse poicionamento e esse alinhamento são alcançados 1 meio de ações coordenadas dos vários grupos musculares responsáveis pela manutenção da relação estabelecida entre os segmentos corporais e da relação do conjunto desses segmentos, ou do corpo como um todo, com o ambiente.

\section{Conclusão}

Sugere-se que o processo de envelhecimento altera o equilíbrio funcional com maior intensidade a partir dos 62 anos de idade.

\section{Referências}

1. Sánches CE, Antolín JCA, Carbajo NF, Carmona RG, López MAL, Juarez AP. Incidência y fatores predictores de inmovilización crónica en ancianos mayores de 75 anos que vivem enla Comunidad. Rev Espanhola de Geriatria y Gerontologia. 2001;36:103-108. 
2. Nóbrega ACL, de Freitas EV, de Oliveira MAB, Leitão MB, Lazzoli JK, Nahas RM, et al. Posicionamento oficial da Sociedade Brasileira de Medicina do Esporte e da Sociedade Brasileira de Geriatria e Gerontologia sobre atividade física e saúde no idoso. Rev Brás Med Esporte. 1999;5(4):207-11.

3. Instituto Brasileiro de Geografia e Estatística - IBGE. Brasil em síntese. 2005. [Acesso em: 22 jun. 2005]. Disponível em: http://www.ibge.gov.br/brasil_em_ sintese/tabelas/populacao_tabela01.htm

4. Silveira CRA, Menuchi MRTP, Simões C'A, Caetano MD, Gobbi LTB. Validade de construção em testes de equilíbrio: ordenação cronológica na apresentação das tarefas. Rev Brás Cineantropom Desempenho Hum. 2006;8(3):66-72.

5. Ramos BMB. Influências de um programa de atividade física no controle do equilíbrio de idosos [dissertação]. São Paulo: Universidade de São Paulo; 2003

6. Tinetti ME, Baker DI, Garrett PA, Gottschalk M, K七 $\Lambda$ ML, Horwitz RI. Yale Ficsit: Risk Factor Abatement Strategy for Fall Prevention. J Am Geriatr Soc. 1993; 41(3):315-20.

7. Tinetti ME, Baker DI, McAvay G, Claus EB, Gá tt P, Gottschalk M, et al. A multifatorial intervention t. duce the risk of falling among elderlv moople living . the community. N Engl J Med. 10 , S3, ):821-7.

8. Hill K, Schwarz J. Assessment manage in older people. Intern Med $\quad 04 ; 34\left(C^{\circ} \quad 0\right): 557-64$.

9. Liu-Ambrose T, Khan KN Eng JJ, Ja n PA, Lord SR, McKay HA. Resistan $r$ and Agility Tra r Reduce Fall Risk in Wome red 75 to 85 with lo bone mass: a 6-mont anu red, control trial. J Am Geriatr Soc. 20 ,52(5):65\%

10. Soares KV, Figueiredo KMOB, Calı is VVA, Guerra RO. Avali o quanto ̀̀ utilização e confiabilidade de instrur itos de $\mathrm{r}$ ida do equilíbrio corporal em idose 'ubllCa. J5;1:78-8'

11. Hawk C, Hy IK, Rupe R, Colonvega M, Hall S. nent of ba. a d risk for falls in a sample of com unity-dwellin adults aged 65 and older. Chirop $+2006 ; 14(3): 1-8$.

Perr ni, MR. Fatores associados a quedas em uma re de idosos residentes no município de São Paùı ce]. São Paulo: Universidade Federal de São Paulo; 20u.
13. Berg KO, Maki BE, Williams JI, Holliday PJ, WoodDauphinee SL. Clinical and oratory sures of postural balance in an eld y population. A Phys Med Rehabil. 1992;73(11 [073-80.

14. Miyamoto ST, Lombardi Ju r I, Berg KO, Ran LR, Natour J. Bazilian version of Berg Balar scale. Braz J Med Biol R

15. Blaszczyk JW ansen PD, Lo DL. Postural sway and percer on the upright s ice stability borders. Percep. 1993;22(11):1 3-41.

16. Blaszczyk JW, Lowe Hans PD. Ranges of postur a ses in the elderly. Gait Po. re. 1. 11-17.

17. Collins De ca CJ, Burrows A, Lipsitz LA. Agerelated $\mathrm{ch}_{\mathrm{s}} \mathrm{s}$ in open-loop and closed-loop postural control me nisms. Exp Brain Res. 1995;104(3): 480-92.

18. eman PA, Leibowitz JM, Blanke D. Age and gender + ts on postural control measures. 1995 Arch Phys Meu Rehabil. 1995;76(10):961-5.

19. Horak FB, Shupert CL, Mirka A. Components of posdyscontrol in elderly: A review. Neurobiol Aging. 1989;10(6):727-38.

20. Woollacott MH, Shumway-Cook A, Nashner LM. Aging and posture control changes in sensory organization and muscular coordination. Int J Aging Hum Dev. 1986; 23(2):97-114.

21. Hay L, Bard C, Fleury M, Teasdale N. Availability of visual and proprioceptive afferent messages and postural control in elderly adults. Exp Brain Res. 1996; 108(1):129-39.

22. Maki BE, Holliday PJ, Fernie GR. Aging and postural control: A comparison of spontaneous- and inducedsway balance tests. J Am Geriatr Soc. 1990;38(1):1-9.

23. Newell KM, Slobounov SM, Slobounova BS, Molenaar PCM. Short-term non-stationarity and the development of postural control. Gait \& Posture. 1997;6(1):56-62.

24. Teasdale N, Stelmach GE, Breunig A, Meeuwsen HJ. Age differences in visual sensory integration. Exp Brain Res. 1991;85(3):691-6.

25. Wolfson L, Whipple R, Derby CA, Amerman P, Murphy T, Tobin JN, Nashner L. A dynamic posturography study of balance in health elderly. Neurology. 1992; 42(11):2069-75. 
26. Dorfman LJ, Bosley TM. Age-related changes in peripheral and central nerve conduction in man. Neurology. 1979;29(1):38-44.

27. Rivner MH, Swift TR, Malik K. Influence of age and height on nerve conduction. Muscle Nerve. 2001; 24(9):1134-41.

28. Maki BE, Mcllroy WE. Postural control in the older adult. Clin Geriatr Med. 1996;12(4):635-58.

29. Spirduso WW. Physical dimensions of aging. Champaign: Human Kinetics; 1995.

30. Häkkinen K, Pastinen UM, Karsikas R, Linnamo V. Neuromuscular performance in voluntary bilateral and unilateral contraction and during electrical stimulation in men at different ages. Eur J Appl Physiol Occup Physiol. 1995;70(6):518-27.

31. Vandervoort AA, Chesworth BM, Cunningham DA, Paterson DH, Rechnitzer PA, Koval JJ. Age and Sex Effects on mobility of human ankle. J Gerontol. 1992: 47(1):M17-21.
32. Young A, Skelton DA. Applied physiolnar of strength and power in old age. Int J Spo vied. I, 5(3): 149-51.

33. Vandervoort, AA. Effects of : ing on human neu muscular function: Implicati for exercise. C Sport Sci. 1992;17(3):178-84.

34. Gatev P, Thomas S pie 1, 'ett M. Feedforward ankle strategy of rance during q. stance in adults. J Physiol. 199r 14( Pt 3):915-28.

35. Gu MJ, Schultz AB, a ard NT, Alex ler NB. Postural control in young and $\mathrm{e}_{\mathrm{r}} \mathrm{v}$ ad $s$ when stance is pertur d. Dynamics. J Biom _ 1996;29(3):319-29.

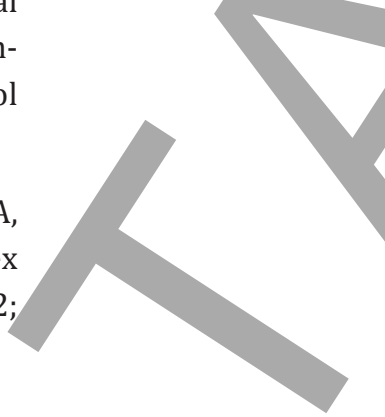

Recebido: $18 / 11 / 2010$

Received: $11 / 18 / 2010$

Aprovado: $14 / 04 / 2011$

Approved: 04/14/2011

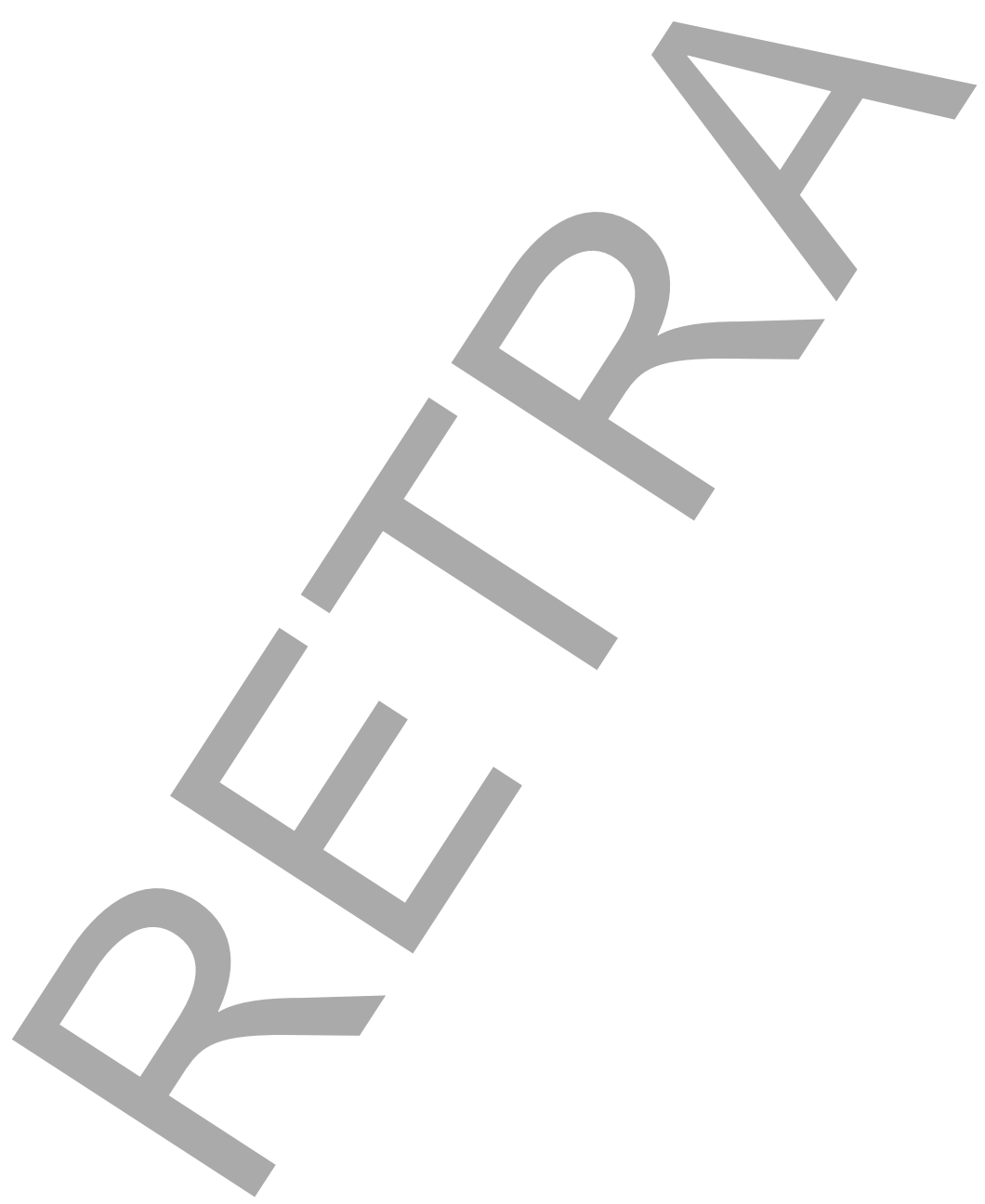

